\title{
DYNAMIC RESPONSE SIMULATION OF PLANETARY GEARS BY THE ITERATIVE SPECTRAL METHOD
}

\author{
Chaari, F.; Hbaeib, R.; Fakhfakh, T. \& Haddar, M. \\ Mechanics Modeling and Production Research Unit \\ Mechanical Engineering Department, National School of Engineers of Sfax, Sfax, Tunisia \\ E-mail: m haddar@voila.fr
}

\begin{abstract}
A planetary gear is modeled in this work. The equations of motion and the eigenfrequencies are recovered. The computation of the dynamic response is made using the spectral iterative method. The procedure is based on a modal approach with developments in the frequency domain. This technique has been successfully used and its convergence was quickly reached. Good agreement was obtained compared with the standard Newmark method. The dynamic response of the planetary gear is given directly in the frequency domain. The inverse Fourier transform gives the time response of the system.
\end{abstract}

Key Words : Planetary Gear, Spectral Method, Dynamic Response Simulation

\section{INTRODUCTION}

Planetary gears are the most used transmission sets in heavy industry such as naval and aeronautic applications that require the transmission of high torque. The modeling of the dynamical behavior of planetary gear was widely treated in literature [1, 2, 3]. All the models include a time varying gearmesh stiffness, which is considered as the main exciting source of the system and is at the origin of the vibration observed $[4,5]$. The computation of the dynamic response in this case couldn't be computed by analytical methods. Classical perturbation methods [6] can be used to solve the problem. However, these methods are essentially based on the assumption of small periodic coefficients, but as in many applications this assumption cannot be justified and these methods are not always valid. Commonly used procedures for finding the response are classical numerical time integration schemes like central-difference, Runge-Kutta and the most known is the Newmark's method [7]. Generally these numerical integration time methods have some disadvantages because of the requirement to eliminate the free response and to choose the integration time step carefully. This is particularly true when the spectral content of the steady state response is broad and marked by both low and high frequencies. For these reasons the computation times can highly increase, in particular when the number of degrees of freedom of the system is high.

Methods based on Fourier series expansion present advantages compared to numerical integration time methods [1]. They do not require the choice of an integration time step, and they allow the error between exact and approximate solutions to be estimated. The spectral iterative method is an extension to these methods [8]. It is based on an iterative scheme developed in the frequency domain.

The spectral method is used to compute the dynamic response of the planetary gear and a comparison with Newmark method is implemented to show the efficiency of this method. 


\section{EQUATIONS OF MOTION OF THE PLANETARY GEAR}

We are treating a problem of plane vibration of a one-stage planetary gear train with $\mathrm{N}$ planets as presented in Fig.1. Sun (s), ring (r), carrier (c) and planets (p) are considered as rigid bodies.

Bearings are modeled by linear springs. The gearmesh is modelled by linear springs acting on the lines of action. Each component has three degrees of freedom: two translations $u_{i}, v_{j}$ and one rotation $w_{j}$, with $w_{j}=r_{j} \Theta_{j}(\mathrm{j}=\mathrm{c}, \mathrm{r}, \mathrm{s}, 1, \ldots \mathrm{N}) ; r_{j}$ is the base radius. Damping is not considered here; nevertheless, it can be introduced in parallel with gearmesh and bearing stiffness.

Radial and tangential coordinates $u_{p}, v_{p}$ describe planet deflections; Translations are measured with respect to the frame $(O, \vec{i}, \vec{j}, \vec{k})$ fixed to the carrier and rotating with a constant angular speed $\Omega_{C}$ in relation to a stationary reference frame. Circumferential planet positions are specified by the fixed angles $\varphi_{p}$ measured relatively to the rotating frame with $\varphi_{1}=0$.

The displacements $\delta_{S p}$ and $\delta_{S p}$ along the lines of action are expressed by [9]:

$$
\begin{gathered}
\delta_{S p}=v_{S} \cos \varphi_{S p}-u_{S} \sin \varphi_{s p}-u_{p} \sin \alpha_{S}-v_{p} \cos \alpha_{S}+w_{S}+w_{p} \\
\delta_{r p}=v_{r} \cos \varphi_{r p}-u_{C} \sin \varphi_{r p}+u_{p} \sin \alpha_{r}-v_{p} \cos \alpha_{r}+w_{r}-w_{p}
\end{gathered}
$$

with $\varphi_{S p}=\varphi_{p}-\alpha_{S}$ and $\varphi_{r p}=\varphi_{p}+\alpha_{r} ; \alpha_{S}$ and $\alpha_{r}$ denotes respectively the pressure angles of sun and ring gears.

Applying Lagrange formulation allow us to recover the equations of motions of the $3 N+9$ degrees of freedom of the system. Assembling the equations in matrix forms leads to the global equation of motion of the system:

$$
M \ddot{x}+\Omega_{C} G \dot{x}+\left[K_{b}+K_{e}(t)-\Omega_{C}^{2} K_{\Omega}\right] x=T
$$

$x$ represents the vector of the degrees of freedom. It is expressed by:

$$
x=\left\langle u_{C}, v_{C}, w_{C}, u_{r}, v_{r}, w_{r}, u_{S}, v_{S}, w_{S}, u_{1}, v_{l}, w_{1}, \ldots, u_{N}, v_{N}, w_{N}\right\rangle^{T}
$$

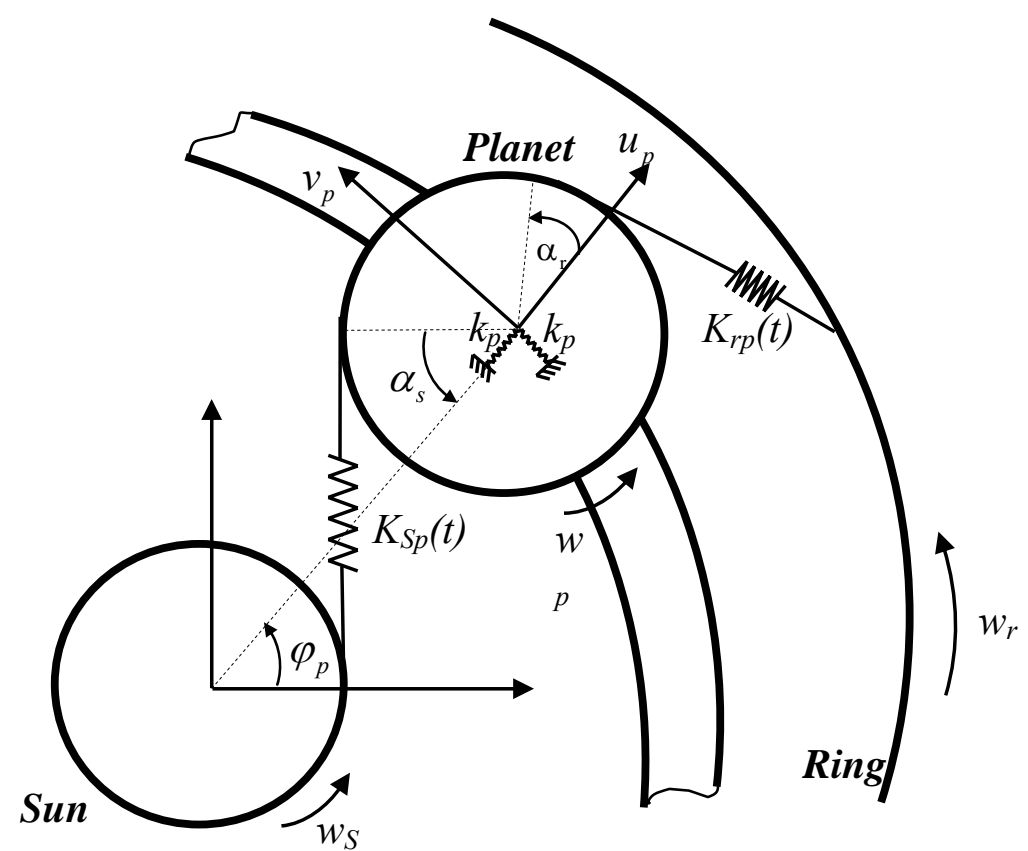

Figure 1: Sun-planet and planet-ring gearmesh modelling. 
$M$ represents the mass matrix. It is expressed by :

$$
M=\left[\begin{array}{cccccc}
M_{C} & 0 & 0 & 0 & 0 & 0 \\
0 & M_{r} & 0 & 0 & 0 & 0 \\
0 & 0 & M_{S} & 0 & 0 & 0 \\
0 & 0 & 0 & M_{1} & 0 & 0 \\
0 & 0 & 0 & 0 & \ddots & \vdots \\
0 & 0 & 0 & 0 & \cdots & M_{N}
\end{array}\right] \text { with: } M_{j}=\left[\begin{array}{ccc}
m_{j} & 0 & 0 \\
0 & m_{j} & 0 \\
0 & 0 & I_{j} / r_{j}^{2}
\end{array}\right] ; \begin{aligned}
& j=c, r, s, 1, \ldots, N ; \\
& \begin{array}{l}
I_{j} \text { is the inertia moment of } \\
\text { the } j \text { th } j^{\text {enent }} \\
\text { to its rotational axis. }
\end{array}
\end{aligned}
$$

The matrix $G$, which is the gyroscopic matrix, can be expressed by:

$$
G=\left[\begin{array}{cccccc}
G_{C} & 0 & 0 & 0 & 0 & 0 \\
0 & G_{r} & 0 & 0 & 0 & 0 \\
0 & 0 & G_{S} & 0 & 0 & 0 \\
0 & 0 & 0 & G_{I} & 0 & 0 \\
0 & 0 & 0 & 0 & \ddots & \vdots \\
0 & 0 & 0 & 0 & \cdots & G_{N}
\end{array}\right] \quad \text { with: } G_{j}=\left[\begin{array}{rrr}
0 & -2 m_{j} & 0 \\
2 m_{j} & 0 & 0 \\
0 & 0 & 0
\end{array}\right] ; j=c, r, s, 1, \ldots, N ;
$$

The bearing stiffness matrix $K_{b}$ is written as:

$K_{b}=\left[\begin{array}{ccc}K_{C b} & 0 & 0 \\ 0 & K_{r b} & 0 \\ 0 & 0 & K_{S b}\end{array}\right]$; where $: K_{j b}=\left[\begin{array}{ccc}k_{j u} & 0 & 0 \\ 0 & k_{j v} & 0 \\ 0 & 0 & k_{j w}\end{array}\right]$;

$k_{j u}, k_{j v}, k_{j w}$ represent the bearing stiffness along the three degrees of freedom. $j=c, r, s$.

The centripetal stiffness $K_{\Omega}$ matrix is expressed by:

$$
K_{\Omega}=\left[\begin{array}{cccccc}
K_{\Omega C} & 0 & 0 & 0 & 0 & 0 \\
0 & K_{\Omega r} & 0 & 0 & 0 & 0 \\
0 & 0 & K_{\Omega S} & 0 & 0 & 0 \\
0 & 0 & 0 & K_{\Omega 1} & 0 & 0 \\
0 & 0 & 0 & 0 & \ddots & \vdots \\
0 & 0 & 0 & 0 & \ldots & K_{\Omega N}
\end{array}\right] \quad \text {; where : } K_{\Omega j}=\left[\begin{array}{ccc}
m_{j} & 0 & 0 \\
0 & m_{j} & 0 \\
0 & 0 & 0
\end{array}\right] .
$$

The gearmesh stiffness matrix is time varying. Generally a square wave form is adopted to express this variation $[6,10]$, the maximum value represents the gearmesh stiffness of two pairs in contact, the minimum one represents single pair gearmesh stiffness. It can be divided into a mean matrix and a time varying matrix:

$$
K_{e}(t)=\bar{K}+K(t)
$$

$K_{e}(t)$ is expressed by : 


$$
K_{e}(t)=\left[\begin{array}{cccccc}
\sum K_{c 1}^{p} & 0 & 0 & K_{c 2}^{l} & \cdots & K_{c 2}^{N} \\
0 & \sum K_{r 1}^{p} & 0 & K_{r 2}^{1} & \cdots & K_{r 2}^{N} \\
0 & 0 & \sum K_{S 1}^{p} & K_{S 2}^{l} & \cdots & K_{S 2}^{N} \\
K_{c 2}^{1} & K_{r 2}^{1} & K_{S 2}^{1} & K^{1} & 0 & 0 \\
\vdots & \vdots & \vdots & 0 & \ddots & 0 \\
K_{c 2}^{N} & K_{r 2}^{N} & K_{S 2}^{N} & 0 & 0 & K^{N}
\end{array}\right]
$$

The components of this matrix are:

$$
\begin{aligned}
& K^{p}=K_{c 3}^{p}+K_{r 3}^{p}+K_{S 3}^{p} ; K_{c 3}^{p}=\left[\begin{array}{ccc}
k_{p} & 0 & 0 \\
0 & k_{p} & 0 \\
0 & 0 & 0
\end{array}\right] ; K_{S 3}^{p}=k_{S p}(t)\left[\begin{array}{ccc}
\sin ^{2} \alpha_{S} & \cos \alpha_{S} \sin \alpha_{S} & -\sin \alpha_{S} \\
\cos \alpha_{S} \sin \alpha_{S} & \cos ^{2} \alpha_{S} & -\cos \alpha_{S} \\
-\sin \alpha_{S} & -\cos \alpha_{S} & 1
\end{array}\right] \\
& K_{r 3}^{p}=k_{r p}(t)\left[\begin{array}{ccc}
\sin ^{2} \alpha_{r} & -\cos \alpha_{r} \sin \alpha_{r} & -\sin \alpha_{r} \\
-\cos \alpha_{r} \sin \alpha_{r} & \cos ^{2} \alpha_{r} & \cos \alpha_{r} \\
-\sin \alpha_{r} & \cos \alpha_{r} & 1
\end{array}\right] \\
& K_{c l}^{p}=k_{p}\left[\begin{array}{ccc}
1 & 0 & -\sin \varphi_{p} \\
0 & 1 & \cos \varphi_{p} \\
-\sin \varphi_{p} & \cos \varphi_{p} & 1
\end{array}\right] ; K_{r l}^{p}=k_{r p}(t)\left[\begin{array}{ccc}
\sin ^{2} \varphi_{r p} & -\cos \varphi_{r p} \cos \alpha_{r} & \sin \varphi_{r p} \\
-\cos \varphi_{r p} \cos \alpha_{r} & \cos ^{2} \varphi_{r p} & \cos \varphi_{r p} \\
\sin \varphi_{r p} & \cos \varphi_{r p} & 1
\end{array}\right] \\
& K_{S 1}^{p}=k_{S p}(t)\left[\begin{array}{ccc}
\sin ^{2} \varphi_{S p} & -\cos \varphi_{S p} \sin \varphi_{S p} & -\sin \varphi_{S p} \\
-\cos \varphi_{S p} \sin \varphi_{S p} & \cos ^{2} \varphi_{S p} & \cos \varphi_{S p} \\
-\sin \varphi_{S p} & \cos \varphi_{S p} & 1
\end{array}\right] \\
& K_{c 2}^{p}=k_{p}\left[\begin{array}{ccc}
-\cos \varphi_{p} & \sin \varphi_{p} & 0 \\
-\sin \varphi_{p} & -\cos \varphi_{p} & 0 \\
0 & -1 & 0
\end{array}\right] ; K_{r 2}^{p}=k_{r p}(t)\left[\begin{array}{ccc}
-\sin \varphi_{r p} \sin \alpha_{r} & \sin \varphi_{r p} \cos \alpha_{r} & \sin \varphi_{r p} \\
\cos \varphi_{r p} \sin \alpha_{r} & -\cos \varphi_{r p} \cos \alpha_{r} & -\cos \varphi_{r p} \\
\sin \alpha_{r} & -\cos \alpha_{r} & -1
\end{array}\right] \\
& K_{S 2}^{p}=k_{S p}(t)\left[\begin{array}{ccc}
\sin \varphi_{S p} \sin \alpha_{S} & \sin \varphi_{S p} \cos \alpha_{S} & -\sin \varphi_{S p} \\
-\cos \varphi_{S p} \sin \alpha_{S} & -\cos \varphi_{S p} \cos \alpha_{S} & -\cos \varphi_{S p} \\
-\sin \alpha_{S} & -\cos \alpha_{S} & 1
\end{array}\right]
\end{aligned}
$$

The external torques applied to the system are: $C_{C}, C_{r}, C_{S}$ respectively on the carrier, the ring and the sun time constant. The corresponding forces are:

$$
T(t)=\left\langle 0,0, T_{C}, 0,0, T_{r}, 0,0, T_{S}, 0, \ldots, 0\right\rangle^{T}
$$

where $T_{j}=C_{j} / r_{j} ; j=c, r, s$.

Modal characteristics of the system are recovered by solving the eigenvalue problem (gyroscopic effect is neglected):

$$
\omega_{i}^{2} M \phi_{i}=\left(K_{b}+\bar{K}\right) \phi_{i}
$$




\section{THE SPECTRAL ITERATIVE METHOD}

In this section the spectral iterative method used to compute the dynamic response is presented. A suitable transformation of (3) is made: the response is divided into a DC component $x_{0}$ and a dynamic component $x_{d}$ :

$$
x=x_{0}+x_{d}
$$

The static response is obtained from the equation:

$$
\tilde{K} x_{0}=T
$$

where $\tilde{K}$ is the mean value of the stiffness matrix of the system expressed by :

$$
\widetilde{K}=K_{b}+\bar{K}-\Omega_{C}^{2} K_{\Omega}
$$

$\bar{K}$ is given by (5).

The contribution of the transmission error will be added later. The excitation is due to time varying gearmesh stiffness. A proportional viscous damping ratio is considered [2].

Rewriting (3) by taking in account the transformation yields to:

$$
M\left(\ddot{x}_{0}+\ddot{x}_{d}\right)+\left(\Omega_{C} G+C\right)\left(\dot{x}_{0}+\dot{x}_{d}\right)+\left[K_{p}+\bar{K}+K(t)-\Omega_{C}^{2} K_{\Omega}\right]\left(x_{0}+x_{d}\right)=T
$$

The dynamic component of the response $x_{d}$ can be represented by the generalized coordinates $q_{d}$, so we can write:

$$
x=x_{0}+\phi q_{d}
$$

Rearranging (12) by the elimination of the static component and taking into account (13) gives:

$$
\phi^{T} M \phi \ddot{q}_{d}+\phi^{T}\left(\Omega_{C} G+C\right) \phi \dot{q}_{d}+\phi^{T}\left(K_{p}+K_{e}(t)-\Omega_{C}^{2} K_{\Omega}\right) \phi q_{d}=-\phi^{T} K(t) x_{0}
$$

Writing (14) in the frequency domain yields to:

$$
\hat{q}_{d}(\omega)=H(\omega)\left[\begin{array}{l}
\hat{t}(\omega)-j \omega \Omega_{C} \sum_{m=1 ; k=1}^{3(N+3)}\left[\phi^{T} G \phi\right]_{m k} \hat{q}_{d k}(\omega) \\
-\sum_{m=1 ; k=1}^{3(N+3)} \int_{-\infty}^{+\infty} e^{-j \omega t}\left[\phi^{T} K(t) \phi\right]_{m k} q_{d k}(t) d t
\end{array}\right]
$$

where: $\hat{q}_{d}(\omega)$ represents the Fourier transform of $q_{d}$ :

$$
H(\omega)=\left(-\omega^{2} \phi^{T} M \phi+\phi^{T} \tilde{K} \phi+j \omega \phi^{T} C \phi\right)^{-1}
$$

$\hat{t}_{j}(\omega)$ represents the Fourier transform of $\phi^{T} K(t) x_{0}$. It is well known that the Fourier transform of the product of two signals $b(t)$ and $c(t)$ in the time domain corresponds to the convolution product of the Fourier transform $\hat{a}$ and $\hat{b}$ of each signal in the frequency domain:

$$
\int_{-\infty}^{\infty} e^{-j \omega t} a(t) b(t) d t=\hat{a}(\omega) \otimes \hat{b}(\omega)
$$

Then (15) can be written as: 


$$
\hat{q}_{d}(\omega)=H(\omega)\left(\hat{t}(\omega)-j \omega \Omega_{C} \phi^{T} G \phi \overline{\hat{q}}_{d}(\omega)-\overline{\hat{K}}(\omega) \otimes \overline{\hat{q}}_{d}(\omega)\right)
$$

$\overline{\hat{K}}(\omega)$ is the Fourier transform of $\phi^{T} K(t) \phi$

The resolution is driven iteratively by successive approximations beginning by an initial solution given by:

$$
\overline{\hat{q}}_{d}^{(0)}(\omega)=H(\omega) \hat{t}(\omega)
$$

The operand $\Delta$ is introduced:

$$
\Delta(\bullet)=-H(\omega)\left(j \omega \Omega_{C} \phi^{T} G \phi(\bullet)+\hat{K}(\omega) \otimes(\bullet)\right)
$$

Applying to (18) we get:

$$
\hat{q}_{d}(\omega)=\hat{q}_{d}^{(0)}(\omega)+\Delta\left(\hat{q}_{d}(\omega)\right)
$$

By successive replacement of $\hat{q}_{d}(\omega)$ and a truncation to order " $h$ " we have:

$$
\hat{q}_{d}(\omega)^{(h)}=\sum_{k=0}^{(+h)} \Delta^{(k)}\left(\hat{q}_{d}^{(0)}(\omega)\right)
$$

where:

$$
\left\{\begin{array}{l}
\Delta^{(0)}\left(\hat{q}_{d}^{(0)}(\omega)\right)=\hat{q}_{d}^{(0)}(\omega) \\
\Delta^{(k+1)}(\bullet)=-H(\omega)\left(j \omega \Omega_{C} \phi^{T} G \phi \Delta^{(k)}(\bullet)+\hat{K}(\omega) \otimes \Delta^{(k)}(\bullet)\right)
\end{array}\right.
$$

The algorithm defining the spectral iterative method is resumed on the following organization chart (next page).

The iterations are stopped and the frequency solution is obtained by reaching an imposed small error " $e r$ " between iterations " $h-l$ " and " $h$ " which is defined by:

$$
e r=\frac{\left|\left(\hat{q}^{h-1}\right)^{2}-\left(\hat{q}^{h}\right)^{2}\right|}{\left(\hat{q}^{h}\right)^{2}}
$$

It is noticed that for reasons of sampling considerations the convolution product is divided by the the number of samples defined by:

$$
M=\frac{T}{\Delta t}
$$

where $T$ is the time duration of the signal and $\Delta t$ is the time step. The spectral response is also divided by $M$.

The time response is obtained by the inverse Fourier transform. 


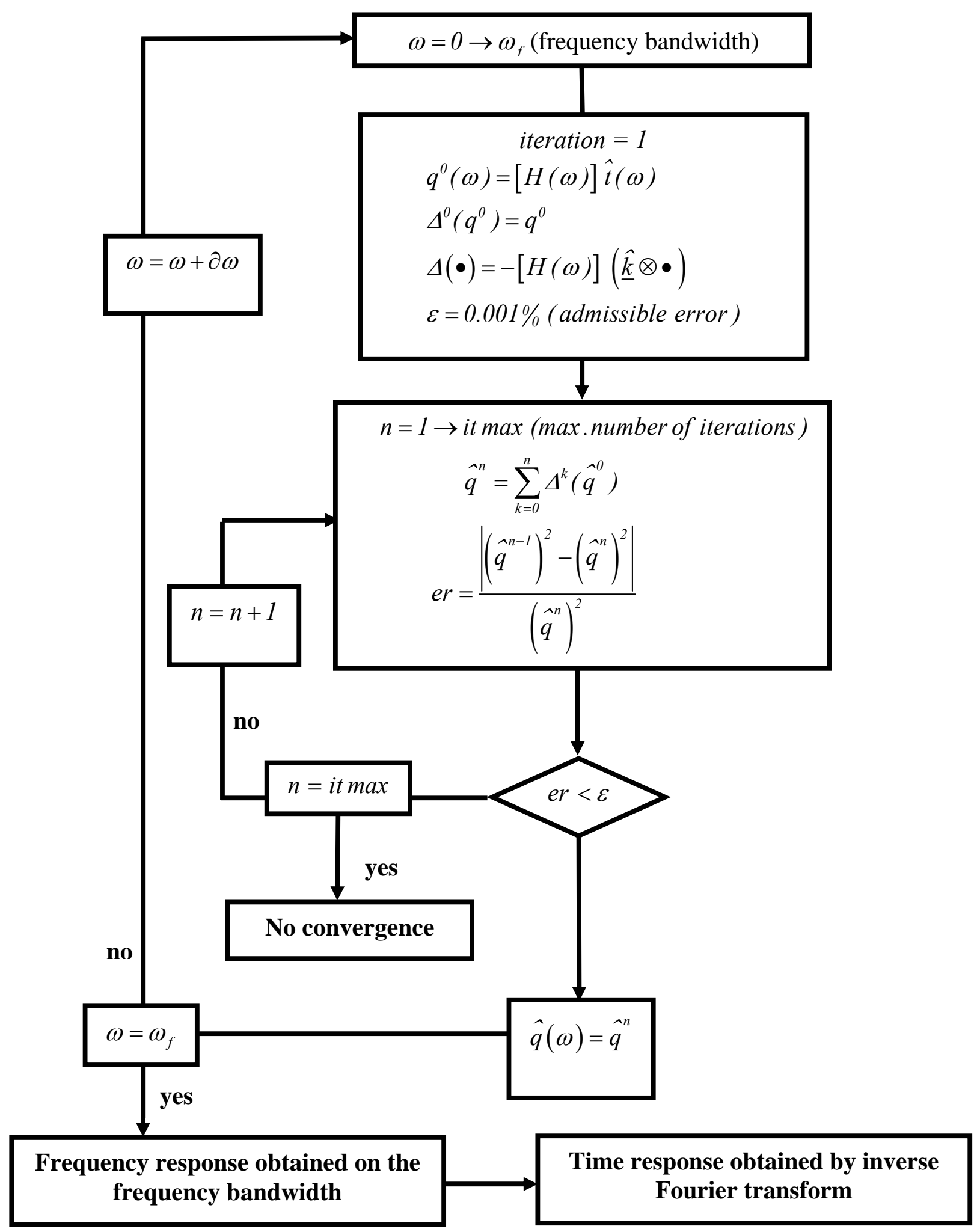

\section{DYNAMIC RESPONSE SIMULATION}

The simulations are made with a planetary gear shown on Fig. 2. An input torque of $1000 \mathrm{Nm}$ is applied to sun gear. The planetary gear, with a fixed ring, has 4 planets. 


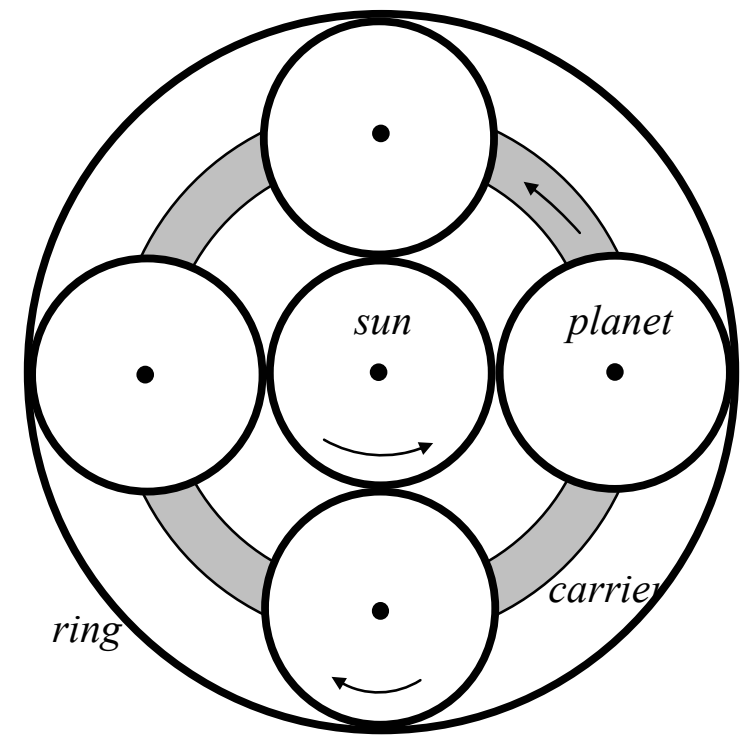

Figure 2: Simulated planetary gear.

The gearmesh frequency $f_{e}$ is defined by:

$$
f_{e}=\frac{Z_{S} Z_{r}}{Z_{S}+Z_{r}} f_{s}
$$

$f_{s}$ represents the sun rotational frequency, $Z_{s}$ and $Z_{r}$ represents respectively the sun and ring tooth number. Parameters of the planetary gear are presented in Table I.

Table I: Parameters of the planetary gear model.

\begin{tabular}{|c|c|c|c|c|}
\cline { 2 - 5 } \multicolumn{1}{c|}{} & Sun & Ring & Carrier & Planet \\
\hline Teeth number & 30 & 70 & -- & 20 \\
\hline module & 1.7 & 1.7 & & 1.7 \\
\hline Teeth width (mm) & 25 & 25 & & 25 \\
\hline Mass (Kg) & 0.46 & 0.588 & 3 & 0.177 \\
\hline $\mathbf{I}^{2} \mathbf{~ ( K g ) ~}$ & 0.272 & 0.759 & 1.5 & 0.1 \\
\hline Base radius (m) & 0.024 & 0.056 & -- & 0.016 \\
\hline Helix angle & $0^{\circ}$ & $0^{\circ}$ & $0^{\circ}$ \\
\hline Gearmesh stiffness (N/m) & \multicolumn{4}{|c|}{$k_{S p}=k_{r p}=2.10^{8}$} \\
\hline Bearing stiffness(N/m) & \multicolumn{5}{|c|}{$k_{r w}=k_{S u, v}=k_{r u, v}=10^{8} ; k_{S w}=k_{C w}=0$} \\
\hline Torsional stiffness (N/m) & \multicolumn{5}{|c|}{$\alpha_{s}=\alpha_{r}=21.34^{\circ}$} \\
\hline Pressure angle & \multicolumn{5}{c}{} \\
\hline
\end{tabular}

The resolution of the eigenvalue problem (6) allows as recovering the eigenfrequencies of the system, which are presented in Table II.

Table II: Eigenfrequencies of the planetary gear.

\begin{tabular}{|c|c|c|c|c|c|c|c|}
\hline & $f_{1}$ & $f_{2}$ & $f_{3}$ & $f_{4}$ & $f_{5}$ & $f_{6}$ & $f_{7}$ \\
\cline { 2 - 9 } Frequency (Hz) & 1099 & 1998 & 2840 & 3165 & 3630 & 3707 & 3938 \\
\cline { 2 - 9 } & $f_{8}$ & $f_{9}$ & $f_{10}$ & $f_{11}$ & $f_{12}$ & $f_{13}$ & \\
\cline { 2 - 8 } & 7998 & 8886 & 9017 & 10490 & 11419 & 12870 & \\
\end{tabular}


The implementation of the spectral iterative method shows a convergence at about the $11^{\text {th }}$ iteration.

Fig. 3 represents the difference proportion between successive iterations for the dynamic component of the rotation of the sun $\left(w_{s}\right)$ and the carrier $\left(w_{c}\right)$ and the radial translation of the first planet $\left(u_{1}\right)$.

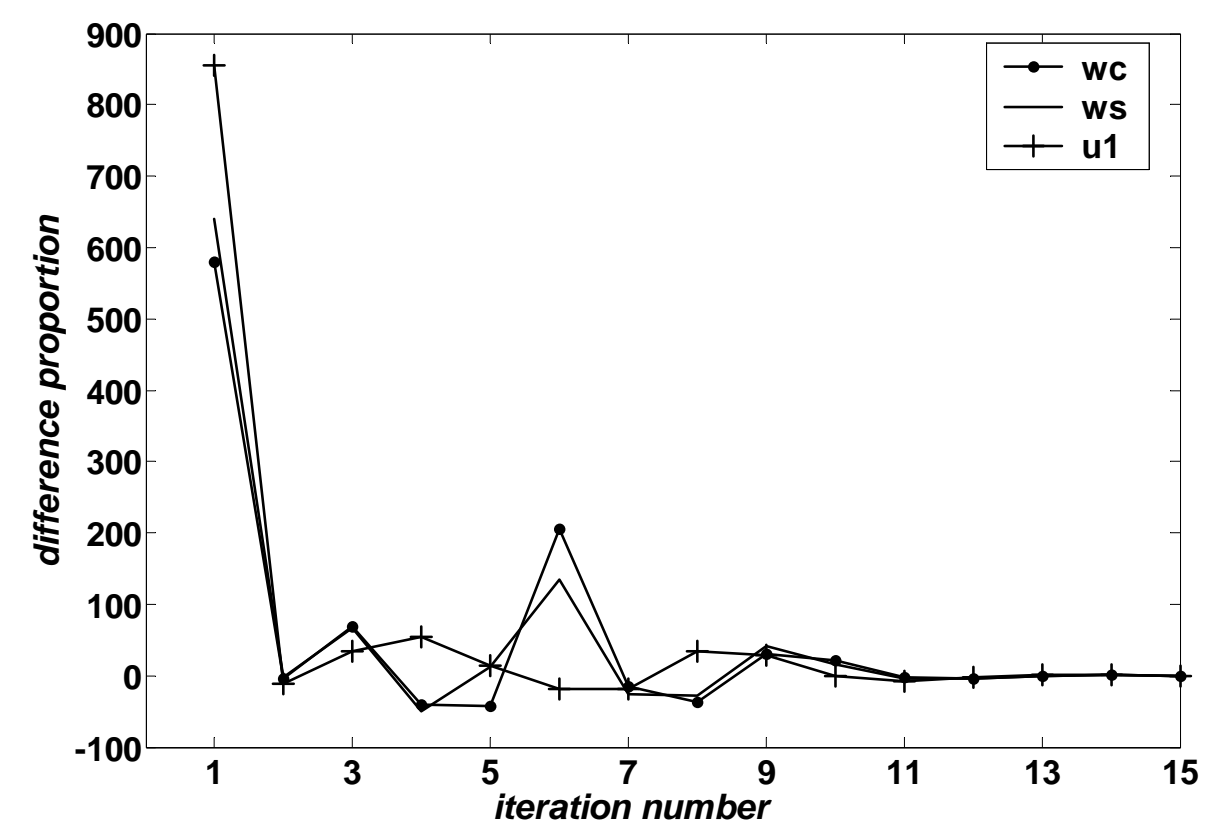

Figure 3: Difference proportion between iterations.

Fig. 4 represents the dynamic coefficient of the sun-planet1 gearmesh for a set of gearmesh frequencies [0-15000 Hz]. This coefficient is defined as the dynamic force divided by the static force. It allows the localization of critical speeds to be avoided.

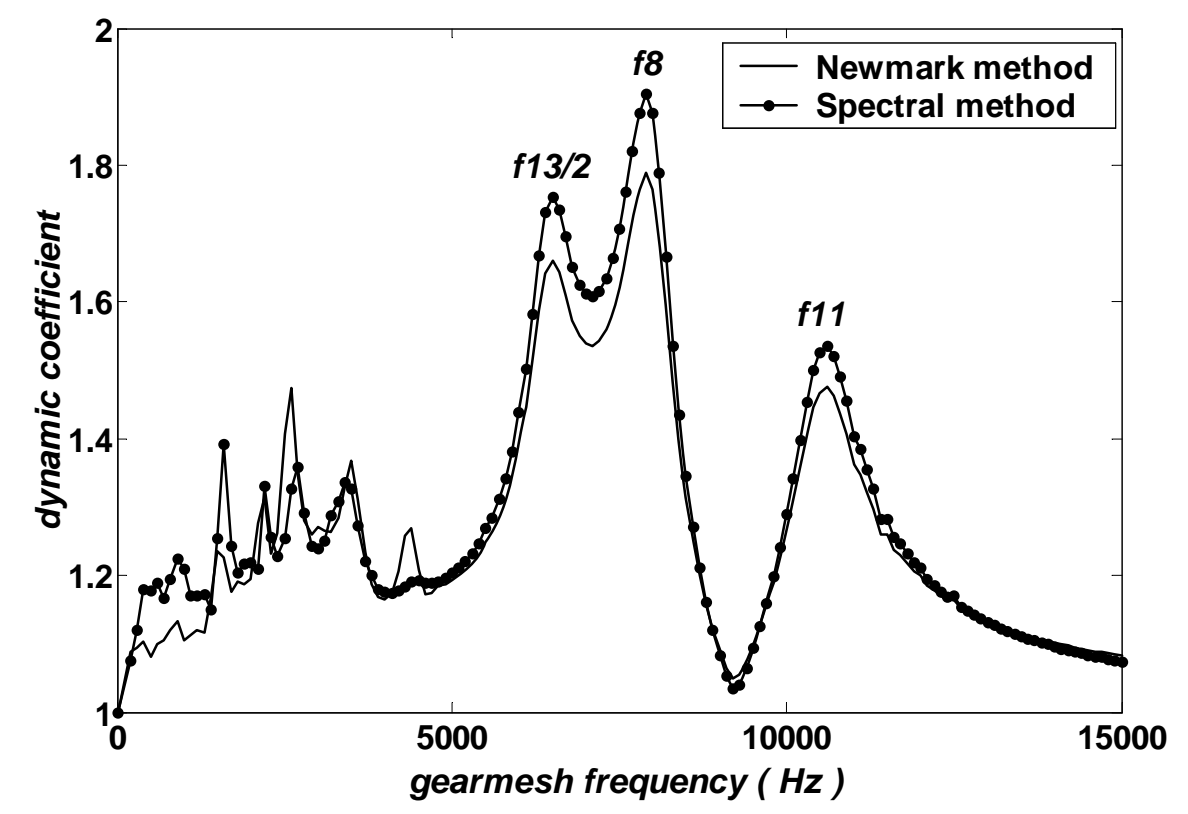

Figure 4: Dynamic coefficient for the sun-planet1 gearmesh. 
Fig. 5 and 6 represent dynamic component of the response registered on the first planet bearing in the radial and tangential directions for the same planetary gear example at $f_{e}=1000 \mathrm{~Hz}$.
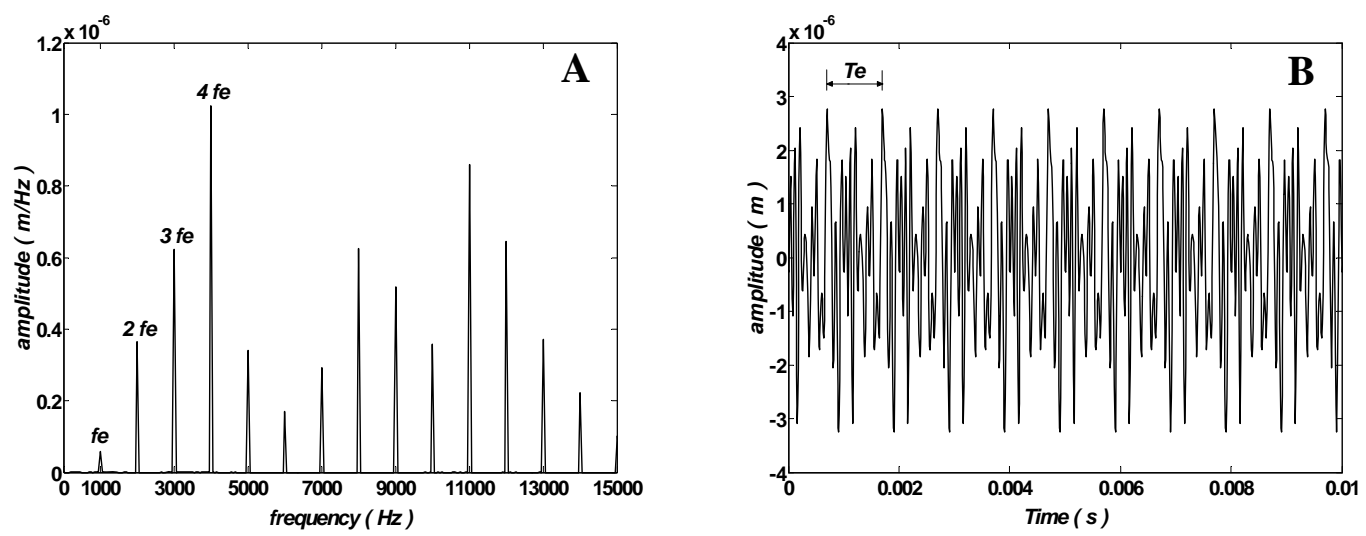

Figure 5: Dynamic component of the radial displacement of the first planet bearing in the frequency domain (A) and in the time domain (B).
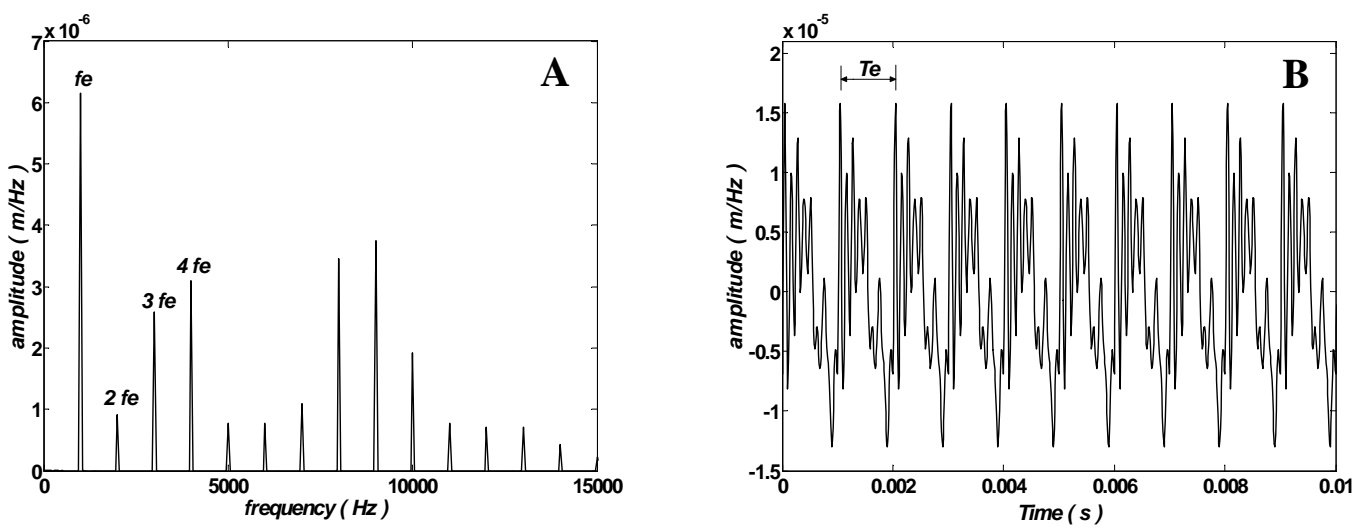

Figure 6: Dynamic component of the tangential displacement of the first planet bearing in the frequency domain $(\mathrm{A})$ and in the time domain $(\mathrm{B})$.

It is well noticed that the response is dominated by the gearmesh frequency $f_{e}$ and its harmonics. The time response is periodic with a period $T_{e}=1 / f_{e}$. The amplitude of the harmonics depends on weather they are close or not to an eigenfrequency.

On the other hand, the computation of the dynamic response by the Newmark and the spectral method showed similar evolutions: the two graphs displayed the same critical frequencies $f_{8}, f_{10} / 2$ and $f_{11}$.

\section{CONCLUSION}

The computation the dynamic response of a planetary gear by the iterative spectral method described in this paper allows one to obtain a direct spectral description of the response. A quick convergence and a good agreement of this method with the Newmark method were obtained. The frequency content of the response is well given and the interactions with eigenfrequencies are also proved. The use of the spectral method can be extended to planetary gear with defects with much frequency content. 


\section{REFERENCES}

[1] Benton, M.; Sereig, A. (1980). The application of the Ritz averaging method to determining the response of systems with time varying stiffness to harmonic excitation, Journal of Mechanical Design, Vol. 102, 384-390

[2] Cunliffe, F.; Smith, J. D.; Welbourn, D. B. (1974). Dynamic Tooth Loads in Epicyclic Gears, Journal of Engineering for Industry, Vol. 94, 578-584

[3] Kahraman, A. (1994). Planetary Gear Train Dynamics, ASME Journal of Mechanical Design, Vol. 116, No. 3, 713-720

[4] Fakhfakh, T.; Chaari, F.; Haddar, M. (2003). Numerical and experimental analysis of gear system with teeth defects, International Journal of Advanced Manufacturing Technology, in press

[5] Hidaka, T.; Terauchi, Y.; Nagamura, K. (1976). Dynamic Behaviour of Planetary Gears 1st Report: Load Distribution, Bulletin JSME, Vol. 19, No. 132, 690-698

[6] Nayfeh, A. H. (1973). Perturbation Methods, New York, John Wiley

[7] Dhatt, G.; Touzot, G. (1984). Une présentation de la méthode des éléments finis, Edition Maloine (in French)

[8] Perret-Liaudet, J. (1996). An original method for computing the response of a parametrically excited forced system, Journal of Sound and Vibration, Vol. 196, 165-177

[9] Saada, A.; Velex, P. (1995). An Extended Model for the Analysis of the Dynamics of Planetary Trains, ASME Journal of Mechanical Design, Vol. 117, 241-247

[10] Chaari, F.; Fakhfakh, T.; Louati, J.; Haddar, M. (2004) Influence of manufacturing errors on the dynamical behavior of planetary gear, International Journal of Advanced Manufacturing Technology, in press 